\title{
A Study of Aerodynamics of Low Reynolds Number Flexible
}

\author{
Airfoils \\ Jian Tang ${ }^{1}$, Dragos Viieru², and Wei Shyy ${ }^{3}$ \\ Department of Aerospace Engineering, \\ University of Michigan, Ann Arbor, MI, 48109, U.S.A.
}

The interaction between aerodynamics and structural flexibility in a low Reynolds number environment is of considerable interest to biological and micro air vehicles. In this study, coupled fluid-structure computations of the Navier-Stokes fluid flow and a flexible airfoil in low Reynolds number environments are conducted to probe the aerodynamic implications. While a flexible airfoil deforms in response to the aerodynamic loading, it exhibits an equivalent pitching motion, which modifies the effective angle of attack, causing noticeable differences in lift and thrust generation. Within the range of the flexibility considered, the flow fields are similar in all cases. Even at $\operatorname{Re}=100$, in the plunging motion, the force acting on airfoil is dominated by pressure and the viscous force is of little impact on the overall lift and thrust generation. Detailed airfoil shape is secondary compared to the equivalent angle of attack.

\section{Nomenclature}

$\begin{array}{ll}c & \text { chord length } \\ C_{x} & \text { horizontal force coefficient }=F_{x} /\left(0.5 \rho c U^{2}\right) \\ C_{y} & \text { vertical force coefficient }=F_{y} /\left(0.5 \rho c U^{2}\right) \\ F_{x} & \text { horizontal force acting on the airfoil } \\ F_{y} & \text { vertical force acting on the airfoil } \\ f & \text { pitching frequency } \\ h(t) & \text { instantaneous position of a plunging airfoil } \\ \mathrm{h}_{\mathrm{a}} & \text { plunging amplitude } \\ p & \text { static pressure } \\ q & \text { load on the beam } \\ \operatorname{Re} & \text { Reynolds number } \\ T & \text { period of one flapping cycle } \\ t & \text { time }\end{array}$

\footnotetext{
1 Post doctoral research fellow, Department of Aerospace Engineering, University of Michigan, jiantang@umich.edu, Member AIAA.

${ }^{2}$ Post doctoral research fellow, Department of Aerospace Engineering, University of Michigan, dviieru@umich.edu, Member AIAA.

${ }^{3}$ Clarence L. "Kelly" Johnson Collegiate Professor and Chair, Department of Aerospace Engineering, University of Michigan, weishyy@umich.edu, Fellow AIAA.
} 


$\begin{array}{ll}U & \text { freestream reference velocity } \\ u, v, w & \text { Cartisian velocity components } \\ x, y, z & \text { Cartesian coordinates } \\ \alpha & \text { angle of attack } \\ \alpha_{0} & \text { initial angle of attack } \\ \alpha_{a} & \text { pitching angle amplitude } \\ \varphi & \text { phase difference between plunging and pitching motion } \\ \mu & \text { coefficient of viscosity } \\ v & \text { kinematic viscosity } \\ \rho_{f} & \left.\text { Fluid density (water density }=1 \times 10^{3} \mathrm{~kg} / \mathrm{m}^{3}\right) \\ \rho_{s} & \text { Structural material density }\end{array}$

\section{Superscript}

non-dimensional quantities

\section{Introduction}

L ATELY, with increasing interest in micro air vehicles and even smaller nano air vehicle, numerous $\boldsymbol{J}$ experimental and numerical research works in the area of aerodynamic force generation by flapping flight have been reported, e.g., ${ }^{1-7}$. The aerodynamic forces in flapping flight predicted by classical, steady state aerodynamic theories ${ }^{1}$ have been found to be insufficient to sustain the insect/birds weight. Recent progress in experimental and computational capabilities have identified four major mechanisms used by insects and birds to generate enhanced lift, namely, Weis-Fogh’s clap-and-fling mechanism² ${ }^{2}$ delayed stall phenomenon associated with leading-edge vortices ${ }^{3}$, fast pitch-up for delayed stall ${ }^{4}$, and wake-capturing for aerodynamics enhancement ${ }^{4}$. These lift generation mechanisms have been identified first experimentally and then confirmed by numerical simulations. For example, Liu and Kawachi ${ }^{5}$ conducted unsteady NavierStokes simulations of the flow around a hawkmoth's wing. Their results showed the leading-edge vortex and the spanwise flow observed experimentally by van den Berg and Ellington ${ }^{6}$ and Ellington et al $^{3}$. Sun and Tang $^{7}$ and Ramamurti and Sandberg ${ }^{8}$ confirmed the force peaks generated during fast pitch-up of the wing at the end of the stroke, and the wake-capturing mechanisms identified experimentally by Dickinson et al. ${ }^{11}$. Wang et al $^{9}$ devised a computational model to solve the Navier-Stokes equation in vorticity-stream function formulation around a two-dimensional moving airfoil. The computed forces of an airfoil undergoing sinusoidal plunging and pitching in a horizontal stroke plane were compared with the threedimensional experimental results of Birch and Dickinson ${ }^{11}$. In the previous efforts, Viieru et al. ${ }^{10}$ and Tang et al. ${ }^{12}$ investigated the lift generation mechanism for a two-dimensional elliptic airfoil undergoing two different hovering motions, one with the airfoil placed horizontally at the end of each stroke (water treading mode $^{13}$ ) and the other with the placed vertically (normal hovering ${ }^{9}$ ). For a Reynolds number of 100 they noticed different force patterns between the two hovering modes suggesting different force generation mechanisms. For the normal hovering mode, a small lift peaks were observed at the beginning of the stroke after the stroke reversal, indicating the presence of a wake-capturing mechanism. Also, for the normal hovering mode as the Reynolds number increases, a distinct asymmetrical force pattern between forward and backward stroke is noticed at small flapping amplitude, as well as changes in the flow field structures. 
In the case of the water treading mode, the lift force variation shows a symmetric pattern between forward and backward stroke. Shyy et al. ${ }^{14}$ have offered a detailed review of the current state of the knowledge in these aspects.

In a recent study, Heathcote et al. ${ }^{17,18}$ investigated the effects of stiffness on thrust generation of an airfoil undergoing a plunging motion under various free stream velocities. Direct force measurements showed that the thrust/input-power ratio was found to be greater for the flexible airfoils than a rigid airfoil. Also they observed that at high plunging frequencies, the medium flexible airfoil generates the largest thrust, while the most flexible airfoil generates the most thrust at low frequencies.

In this paper we investigate the flapping motion in a uniform stream that mimics the forward insect flight. Specifically, we investigate the airfoil of varying degrees of flexibility under different Reynolds number (9,000 and 100). Specifically, we probe the following questions:

1. How does the structural flexibility affect the fluid flow around an airfoil undergoing a plunging motion?

2. How does the combined structural flexibility and plunging motion affect the geometry of the airfoil and the effective angle attack?

3. How do the above two aspects affect the aerodynamic outcome, namely, the lift and thrust generation?

4. What roles do the pressure and viscous effects play in affecting a flexible, plunging airfoil's aerodynamic performance?

The parameters adopted in the present study are relevant, but not identical, to those in the experimental work of Heathcote and Gursul ${ }^{18}$. In this study our main interest is to assess the above issues qualitatively, with more detailed quantitative investigations to follow in the near future. To study the effect of the flexibility, a finite element model using beam elements is employed. The structural solver is coupled with the flow solver by inner iteration at each time step. A pressure-based flow solver, along with a structured grid redistribution algorithm, capable of handling large translations and rotations characteristics of deform motion, is developed and employed in coupling with the structure solver. The flow-structure coupling is realized by inner iteration in each time step.

\section{Numerical Algorithm}

\section{A. Flow solver}

The fluid dynamics are solved from the incompressible Navier-Stokes equations and the continuity equation, written as

$$
\begin{aligned}
\frac{\partial u_{i}}{\partial t}+\frac{\partial}{\partial x_{j}}\left(u_{j} u_{i}\right) & =-\frac{1}{\rho_{f}} \frac{\partial p}{\partial x_{i}}+v \frac{\partial^{2} u_{i}}{\partial x_{j}^{2}} \\
\frac{\partial u_{j}}{\partial x_{j}} & =0
\end{aligned}
$$


where $\rho_{f}$ is the fluid density, $u_{i}$ is the velocity vector, $t$ is the time, $x_{i}$ is the position vector, $p$ is the pressure, and $v$ is the kinematic viscosity.

The flapping kinematics is described as follows. A schematic of the plunging displacement $h(t)$ and pitching angle $\alpha(t)$ for the plunging motion mode are shown in Fig. 1. The plunging and pitching of the upward- and downward-strokes in each cycle are the sinusoidal function. The incoming flow is along the horizontal direction. Specifically, the plunging and pitching amplitudes are:

$$
\begin{aligned}
& h(t)=h_{a} \sin (2 \pi f t) \\
& \alpha(t)=\alpha_{0}+\alpha_{a} \sin (2 \pi f t+\varphi)
\end{aligned}
$$

where $\alpha_{a}$ is the pitching amplitude, $h_{a}$ is the dimensionless plunging amplitude normalized by the chord length $c, \alpha_{0}$ is the initial positional angle of the airfoil, and $\varphi$ is the phase difference between the plunging and pitching motion. Also, $h(t)=y_{L E}(t) / c$ where $y_{L E}$ is the vertical coordinate of the leading edge of the airfoil. In the present investigation, two kinematic patterns are considered, namely, (i) forward flight with pure plunging and no pitching, and (ii) forward flight with combined plunging with pitching.

For forward flight, the freestream velocity $(U)$, the chordlength $(c)$ and the inverse plunging/pitching $(1 / f)$ frequency are used as the velocity, length and time scales, respectively. Consequently, the Reynolds and Strouhal numbers appear, i.e., $\operatorname{Re}=U c / v$, and $S t=f c / U$. With these choices of the scaling parameters, the non-dimensional form of the Navier-Stokes equations become

$$
\text { St } \frac{\partial}{\partial \bar{t}}\left(\bar{u}_{i}\right)+\frac{\partial}{\partial \bar{x}_{j}}\left(\bar{u}_{j} \bar{u}_{i}\right)=-\frac{\partial \bar{p}}{\partial \bar{x}_{i}}+\frac{1}{\operatorname{Re}} \frac{\partial^{2} \bar{u}_{i}}{\partial \bar{x}_{j}^{2}}
$$

An additional non-dimensional parameter is introduced following the definition by Heathcote and Gursul ${ }^{18}$. For hovering, since the freestream velocity is zero, the Reynolds number is defined as $\operatorname{Re}_{f}=f c^{2} / v$. This Reynolds number is a combination of $\mathrm{Re}$ and $\mathrm{St}$ as $\mathrm{Re}_{f}=\mathrm{St} \cdot \mathrm{Re}$.

It is noted that in the literature, the Reynolds number for hovering flight is often defined based on the maximum velocity $U_{\max }=2 \pi f y_{\max }$. 


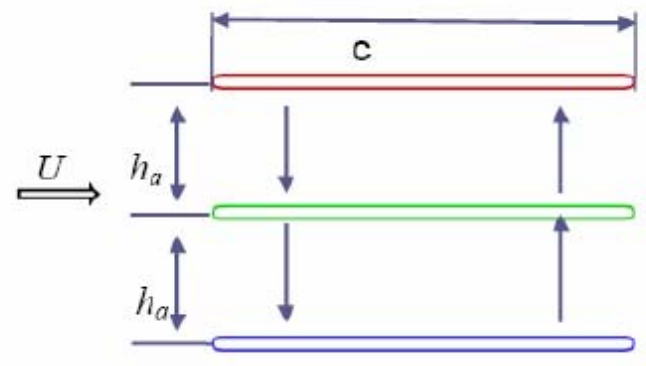

(i) plunging mode

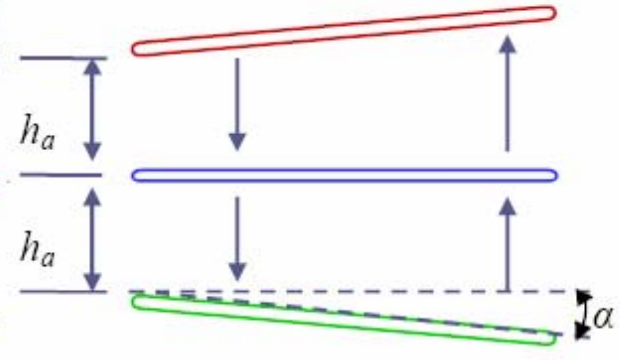

(ii) plunging and pitching mode

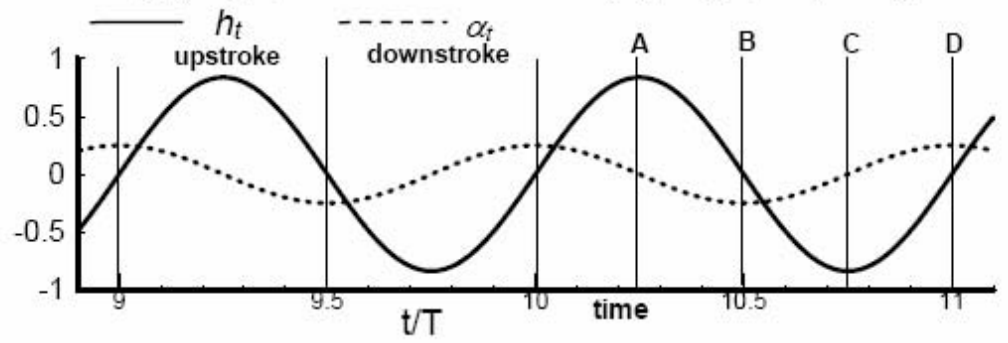

(iii) time history of plunging and pitching motion

Fig. 1 Illustration of the flapping motion. (i. plunging mode; ii. plunging and pitching mode; iii. time history of pitching and plunging motion).

A pressure-based Navier-Stokes solver on the structured moving grid is employed in this study. The flow solver is based on the extension of the original SIMPLE family of algorithms ${ }^{19}$, with an employment of the Cartesian and contravariant velocity variables to facilitate strong conservation law formulations and consistent finite volume treatment ${ }^{19,20}$. The convection terms are discretized using a second-order upwind scheme $^{20}$, while for the pressure and viscous terms a second-order central difference scheme is used. For time integration, an implicit second order upwind scheme is employed. To handle the pitching motion and deformation of the airfoil, an algebraic grid generating technique is employed at each time step. The algebraic grid generator generates a new grid based on the deformed airfoil in each time step with affordable extra computing expense. The geometric conservation law (GCL) originally proposed by Thomas and Lombard ${ }^{21}$ was incorporated to consistently compute the cell volume in a moving boundary problem and eliminate the artificial mass sources. The specific implementation and implications of the GCL in the context of the present solution algorithm have been discussed by Shyy et $\mathrm{al}^{22}$.

\section{B. Structural solver}

To solve for the structural displacements, we propose a 2D finite element method using beam elements. The displacement $y$ is given by the beam differential equation

$$
\rho_{s} b \frac{\partial^{2} y}{\partial t^{2}}+\frac{\partial^{2}}{\partial x^{2}}\left(E I \frac{\partial^{2} y}{\partial x^{2}}\right)=q
$$


where $\rho_{s}$ is the density of the beam, $b$ is the beam height, $E$ is the Young's modulus, $I$ is the area moment of inertia of the cross section of beam element, and $q$ is the distributed loading acting in the same direction of the displacement $y$.

Non-dimensionalizing by $c, \rho$, and $U$, we obtain

$$
\bar{\rho} \frac{b}{c} \frac{\partial^{2} \bar{y}}{\partial \bar{t}^{2}}+\overline{E I} \frac{\partial^{4} \bar{y}}{\partial \bar{x}^{4}}=\bar{q}
$$

where $\left(^{-}\right.$) denotes again the non-dimensionalized variables. The non-dimensional parameters, density $\bar{\rho}$, modulus of elasticity $\bar{E}$ and inertia area moment of the beam cross section $\bar{I}$, are defined as

$$
\begin{aligned}
& \bar{\rho}=\frac{\rho_{s}}{\rho_{f}} \\
& \bar{E}=\frac{E}{\rho_{f} U^{2}} \\
& \bar{I}=\frac{I}{c^{3}}
\end{aligned}
$$

For a flat plate with thickness $b$ and unit width, the area moment of inertia is $I=1 / 12 \cdot b^{3}$. Then the bending rigidity becomes

$$
\bar{I}=\frac{1}{12}\left(\frac{b}{c}\right)^{3}
$$

Since the Young's modulus $E$, the densities $\rho_{f}, \rho_{s}$ and velocity scale $U$ are considered to be constant, a representative non-dimensional parameter is then the relative to thickness $b / c$. The coupling procedure in each time step for the fluid-structure problem is iterated as following: 


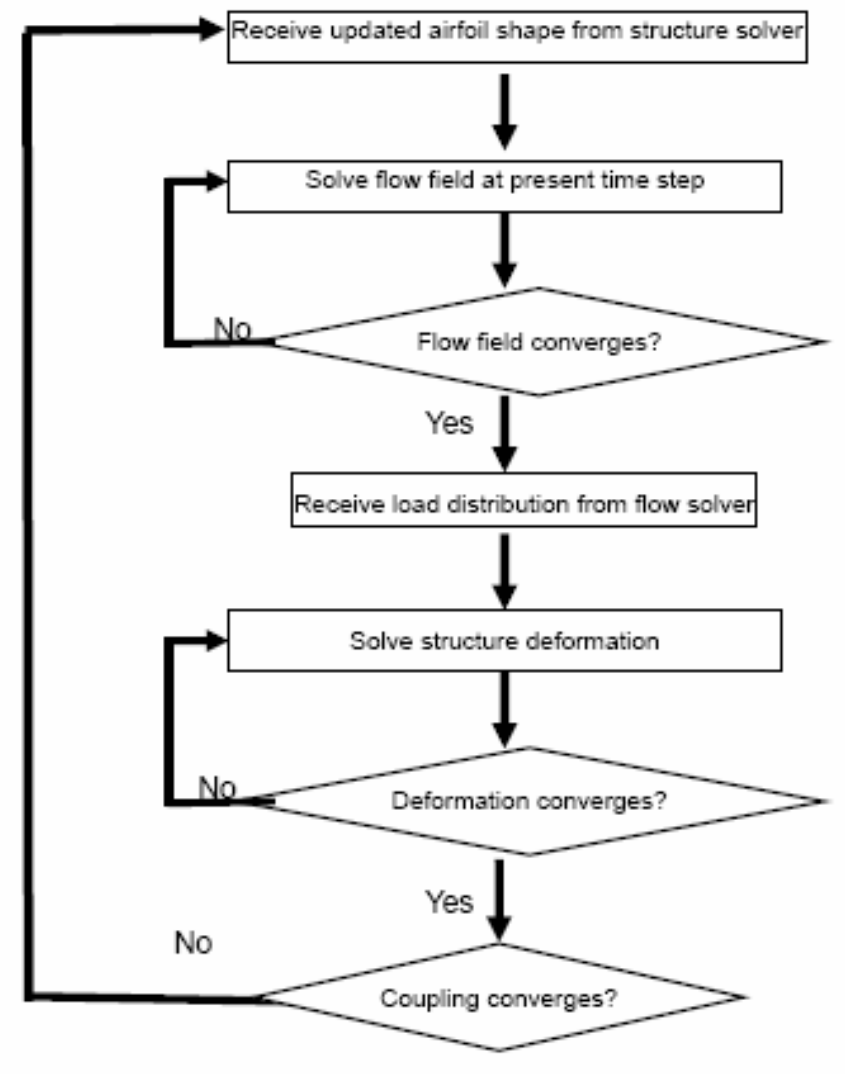

Fig. 2 Flow chart of the flow-structure coupling procedure.

(i) Receive the new airfoil shape from the structure solver (in the first iteration, use previous time step airfoil shape)

(ii) Solve the flow field based on the updated airfoil shape; sub-iteration is used in the flow solver before reaching convergent limit.

(iii) Transfer the load from flow structure solver to the structure solver.

(iv) Solve the structural deformation by sub-iteration. The iteration is stopped after the deformation convergent limit is reached.

(v) If the difference between present and previous deformation is less than the convergent limit, stop iteration.

In summary, the key dimensionless parameters result from Eqs. (3) and (5) are Re, St, $\bar{E}$, and b/c. While one can choose to use different time step sizes in the fluid dynamics and structural dynamics modules, in the present study, the physical time steps are identical between fluids and structures solvers.

\section{Results and Discussion}

Utilizing the computational algorithm introduced above, two cases are investigated. 
(i) coupled fluid flow and flexible structural dynamics: the fluid flow around the plate of different thickness with tear-drop-shaped leading edge are solved at Reynolds number 9000;

(ii) fluid flow around an airfoil with prescribed shape change: a flat plate with half cylinders at leading and trailing edges are investigated at Reynolds number 100 to probe the mechanism of the thrust generation.

In the following computation, two grids are utilized corresponding to the airfoil introduced above. The two grids are both O-type with 1\% thickness relative to the chord length. The grid of the plate with a teardrop element at the leading edge (11.1\% relative to the chord length) is shown in Fig. 3 . The distance from the solid wall to the first grid point is $10^{-4}$ with outer boundary locating at 16 chord length from the center (Fig. 3). The grid size of the flat plate with half cylinders at leading and trailing edges is shown in Fig. 3. The distance from the solid wall to the first grid point is $10^{-3}$ with outer boundary locating at 16 chord length from the center.
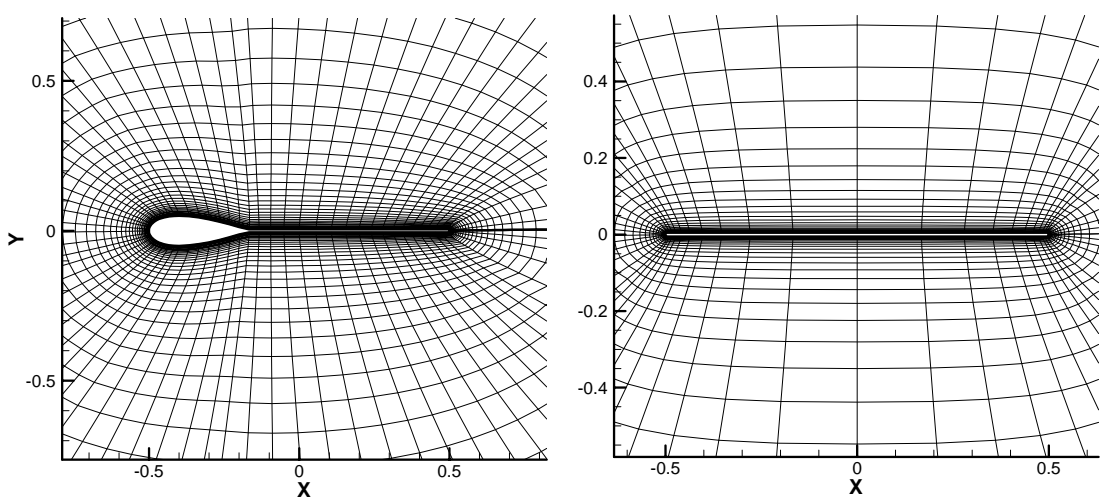

Fig. 3 Grid distribution of the flat plates (left: flat plate with a tear-drop element at the leading edge, right: flat plate with half cylinders at leading and trailing edges).

In the following cases, sinusoidal function (Eq. 2) is employed in both plunging and pitching motions in the numerical simulation. Pitching motion is synchronized with the plunging motion with a fixed phase lag $\pi / 2$. The center of pitching motion is at the leading edge of the airfoils. The parameters used in the following are defined by:
$h(t)=y_{L E}(t) / c$
$\operatorname{Re}=U c / v$
$\operatorname{Re}_{f}=f c^{2} / v$
$S t=f c / U$

where, $y_{L E}(t)$ is the vertical coordinate of the leading edge of the airfoil, the reference velocity is the incoming velocity $U$. Please note that $S t=\operatorname{Re}_{f} / \mathrm{Re}$ in the present parameters definition. The present flow solver coupled with the moving mesh algorithm is validated, in previous studies (Tang, Viieru and Shyy ${ }^{12}$ ), by comparing the current result for the normal hovering mode at a Reynolds number of 75 with the computational results of Wang et al. ${ }^{9}$ and the experimental results of Birch, Dickson and Dickinson ${ }^{11}$. The results showed a good agreement with the experimentally measurements.

\section{A. Forward Flight with Flexible Plunging Airfoil}


Flow field is investigated at the $\mathrm{Re}=9000$. Non-dimensional material properties are given as: modulus of elasticity $2.05 \times 10^{10}$, density 7.85 . The stiffness varies by changing the thickness of the plate. Three plates with relative thickness of $\mathrm{b} / \mathrm{c}=0.56 \times 10^{-3}, \mathrm{~b} / \mathrm{c}=1.41 \times 10^{-3}, \mathrm{~b} / \mathrm{c}=4.23 \times 10^{-3}$ are used in present research. The airfoil performs plunging motion without pitching $\left(\alpha_{a}=0, \alpha_{0}=0\right.$ ). The plunging amplitude is $h_{a}=0.194$, normalized by the chord length $c$, Strouhal number is 1.4 , and $\varphi=\pi / 2$. Differences between the leading and trailing edge's vertical coordinates, together with the position of the leading edge are presented in Fig. 4. The results indicate that deformation not only increase with the flexibility of the plate, which is obvious, but also creates a phase difference relative to the pitching motion. To characterize the deformation of the airfoil, an equivalent pitching angle is adopted, which is defined as the pitching angle of a rigid airfoil with same leading and trailing edge positions of the flexible airfoil. Considering that the length of plates does not change, the vertical coordinates' difference reflects the equivalent pitching angle. Fig. 4 indicates that the equivalent pitching motion of a flexible plate (LE-TE, $b / c=0.56 \times 10^{-3}$ ) leads the plunging motion (position history of leading edge). This observation agrees with the experimental work of Heathcote and Gursul ${ }^{18}$.

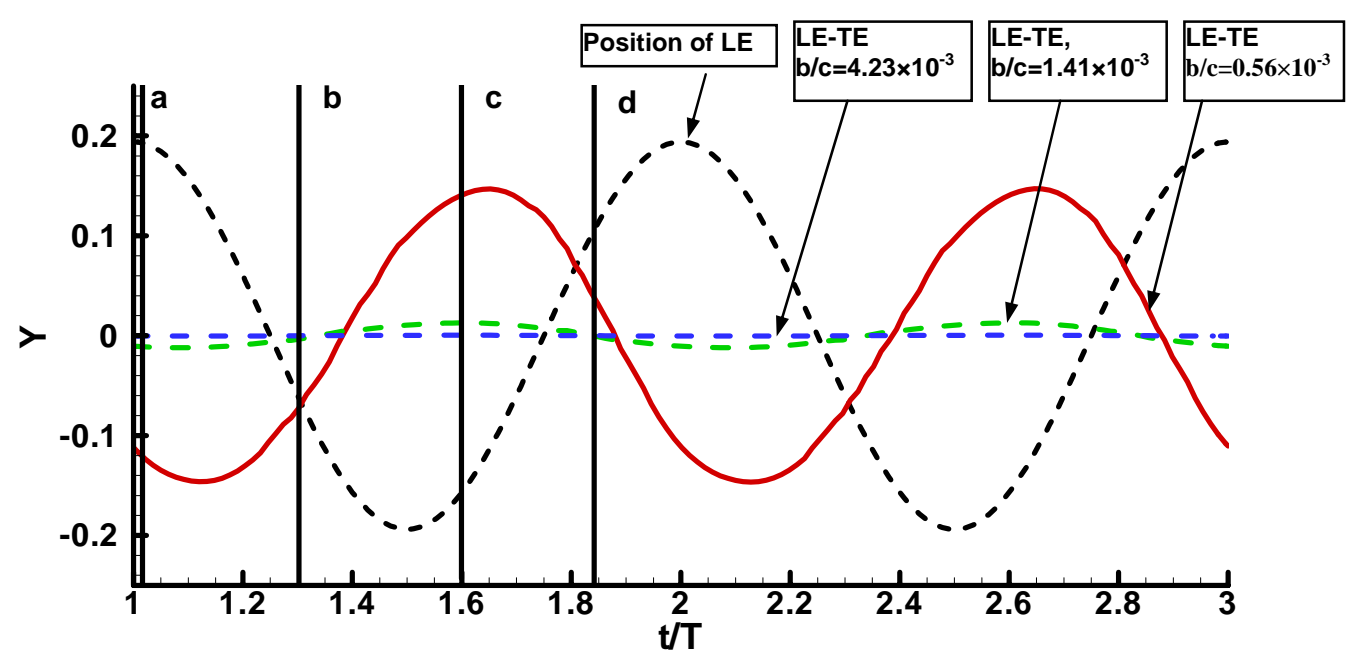

Fig. 4 Time history of displacement differences of $\mathrm{LE}$ and $\mathrm{TE}$ coordinate $\left(\mathrm{Re}=9000, S t=1.4, \mathrm{~h}_{\mathrm{a}}=\mathbf{0 . 1 9 4}\right.$, $\mathrm{b} / \mathrm{c}=0.56 \times 10^{-3}, \mathrm{~b} / \mathrm{c}=1.41 \times 10^{-3}, \mathrm{~b} / \mathrm{c}=4.23 \times 10^{-3}$. LE: leading edge, TE: trailing edge).

Fig. 5 further illustrates the effect of the equivalent pitching angle by presenting the effective angle of attack, which is based on the rigid airfoil's instantaneous position in accordance with the combined free stream and plunging motion, and equivalent pitching angle vs. time. It indicates that as the airfoil becomes more flexible, the effective angle of attack is reduced by the equivalent pitching motion. This means that for a flexible airfoil, the lift fluctuation during a flapping cycle is smaller than for a rigid airfoil. Similar observations have been reported for fixed wing, where a flexible structure can smooth out the fluctuation in lift in a gusty free stream ${ }^{14,15}$. 


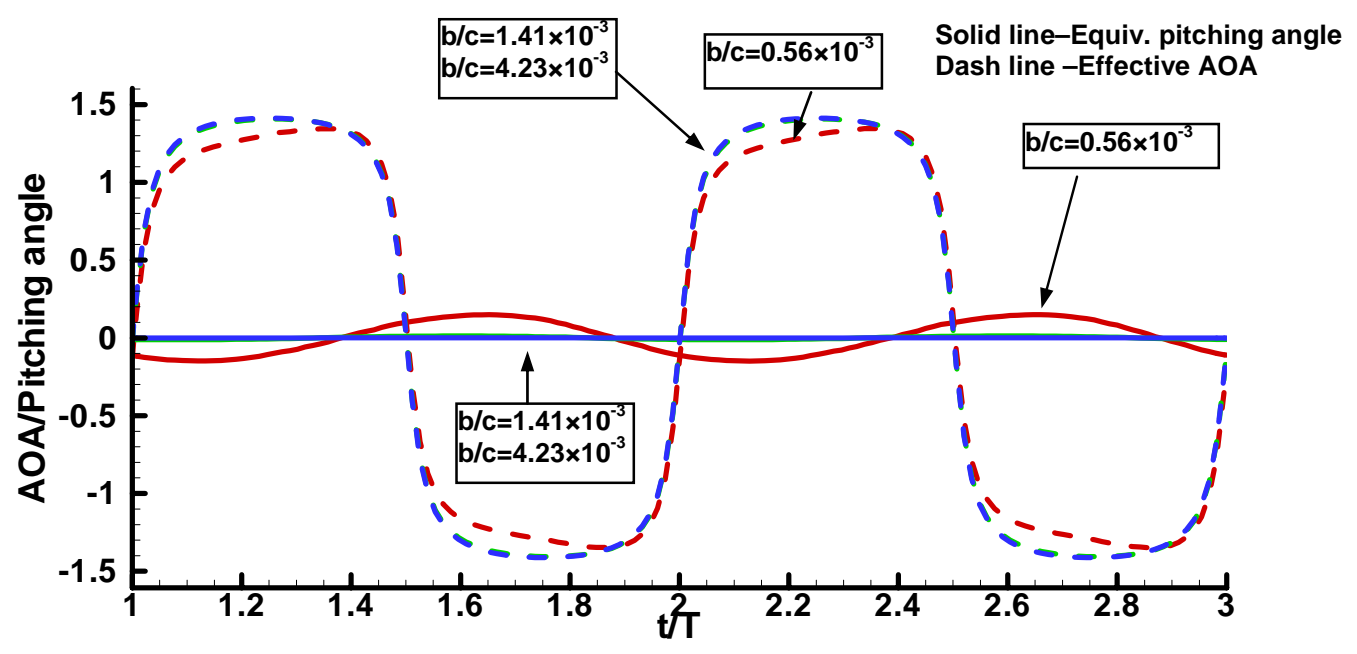

Fig. 5 Time history of equivalent pitching angle and effective $A O A\left(R e=9000, S t=1.4, h_{a}=0.194\right.$, $b / c=0.56 \times 10^{-3}, b / c=1.41 \times 10^{-3}, b / c=4.23 \times 10^{-3}$ ).

Fig. 6 presents the time history of the horizontal (thrust) and vertical (lift) force coefficients. It shows that as the airfoil becomes more flexible, a higher thrust and, a smaller lift, is generated.
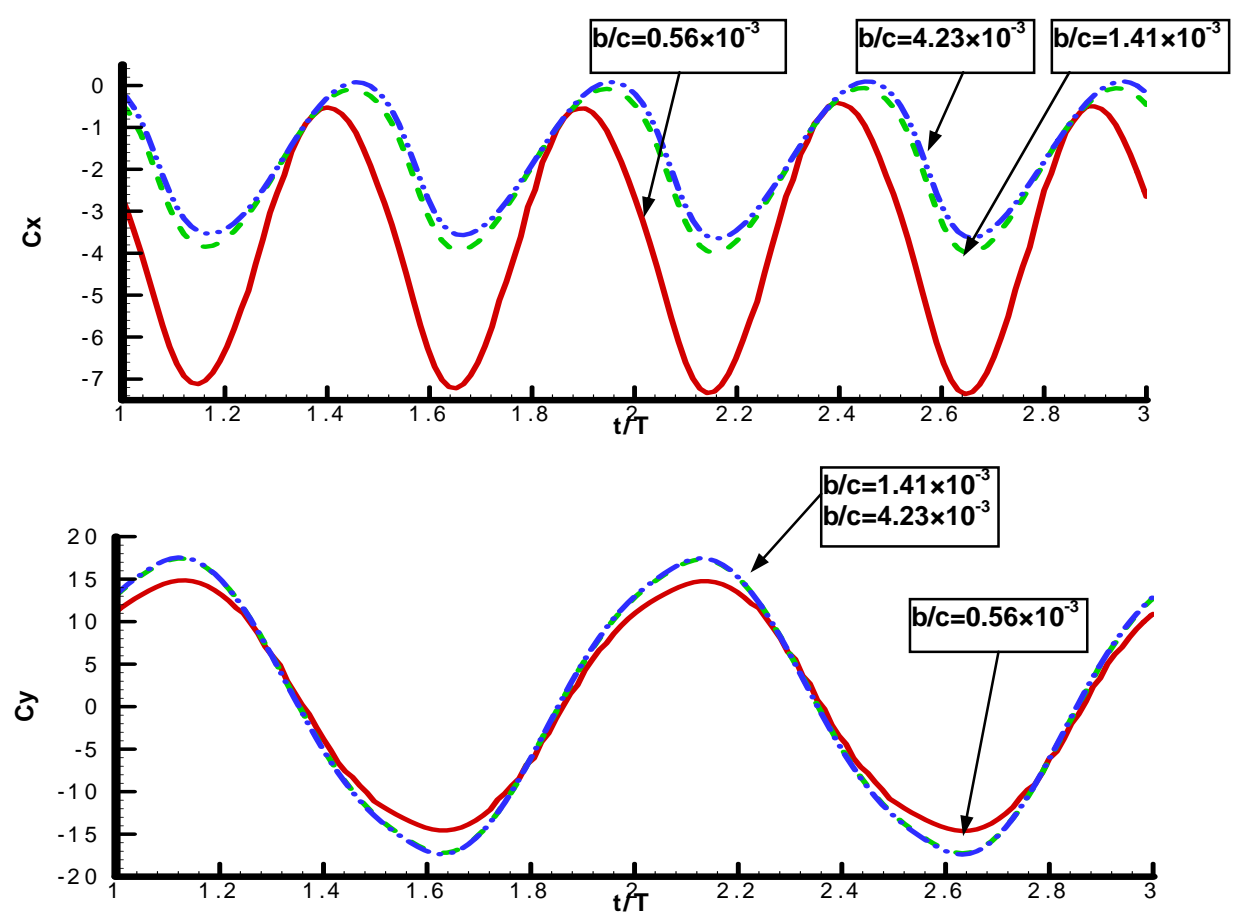

Fig. 6 Time history of coefficients of horizontal and vertical force $\left(R e=9000, S t=1.4, h_{a}=0.194\right.$, and three thicknesses: $b / c=0.56 \times 10^{-3}, b / c=1.41 \times 10^{-3}, b / c=4.23 \times 10^{-3}$ ). 
Fig. 7 presents the streamlines, pressure contours and surface pressure distributions around the airfoil with different thicknesses at the time instants indicated in Fig. 4. Overall, the fluid dynamics characteristics of all three cases are substantially the same. However, Fig. 6 shows that the horizontal force (thrust) on the thinnest plate is much larger than the two stiffer plates. It seems that the pitching motion resulting from the airfoil shape flexibility impacts the equivalent angle of attack, causing different projected horizontal and vertical areas, and correspondingly, different lift and thrust coefficients. To further study the interplay between similar flow field and different force coefficients exhibited by airfoils of different flexibility, a numerical experiment is conducted in the next section.

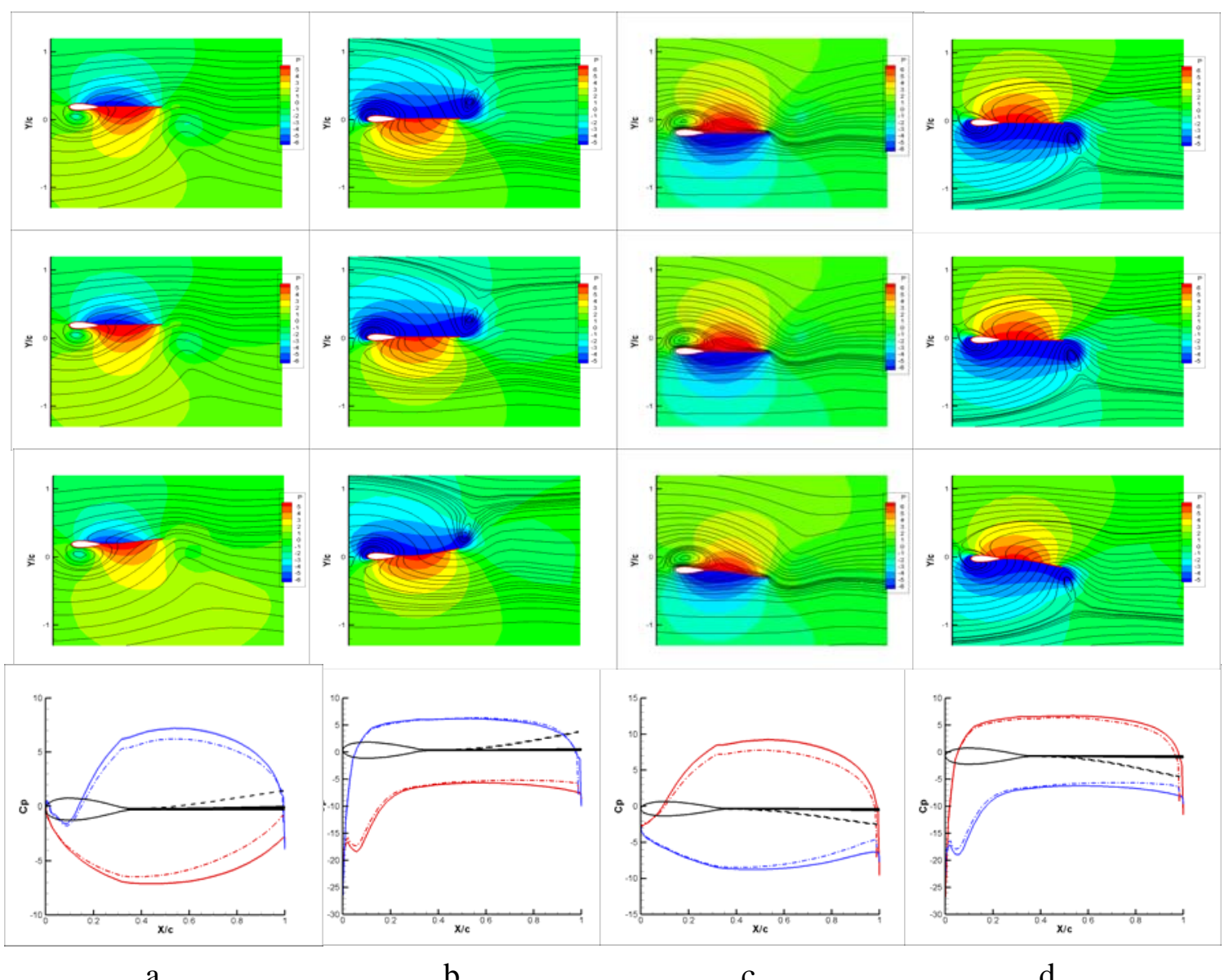

Fig. 7 Flowfield of the numerical results of three thickness plates $\left(\operatorname{Re}=9000, S t=1.4, b / c=4.23 \times 10^{-3}\right.$ (top), $b / c=1.41 \times 10^{-3}$ (middle), $b / c=0.56 \times 10^{-3}($ lower $)$ ) and the pressure distribution on the airfoils (bottom) (in pressure distribution, solid lines (overlapped): $b / c=4.23 \times 10^{-3}, b / c=1.41 \times 10^{-3}$; dash line: b/c $\left.=0.56 \times 10^{-3}\right)$.

\section{B. Assessment of the Shape Change to the Aerodynamics with Prescribed Deformation}

To investigate the effect of the flexibility, a numerical experiment is conducted to study the effect of deformation at low Reynolds number at $\mathrm{Re}=100$. Part of the reason to choose a lower Reynolds number here is to probe both the implications of the airfoil shape change on the aerodynamics, and the roles between pressure and viscous effects associated with a plunging airfoil. Specifically, the plunging amplitude is $h_{a}=0.194, \mathrm{St}=0.3 . \quad \alpha_{0}=0, \alpha_{a}=0$ and $\varphi=\pi / 2$. We set one case with prescribed deformation simulating the flexible airfoil and another one performing the same pitching and plunging 
motion but with no shape change. The displacements of the trailing edges are set exactly the same in these two cases to test the difference in thrust generation (shown in Fig. 8).

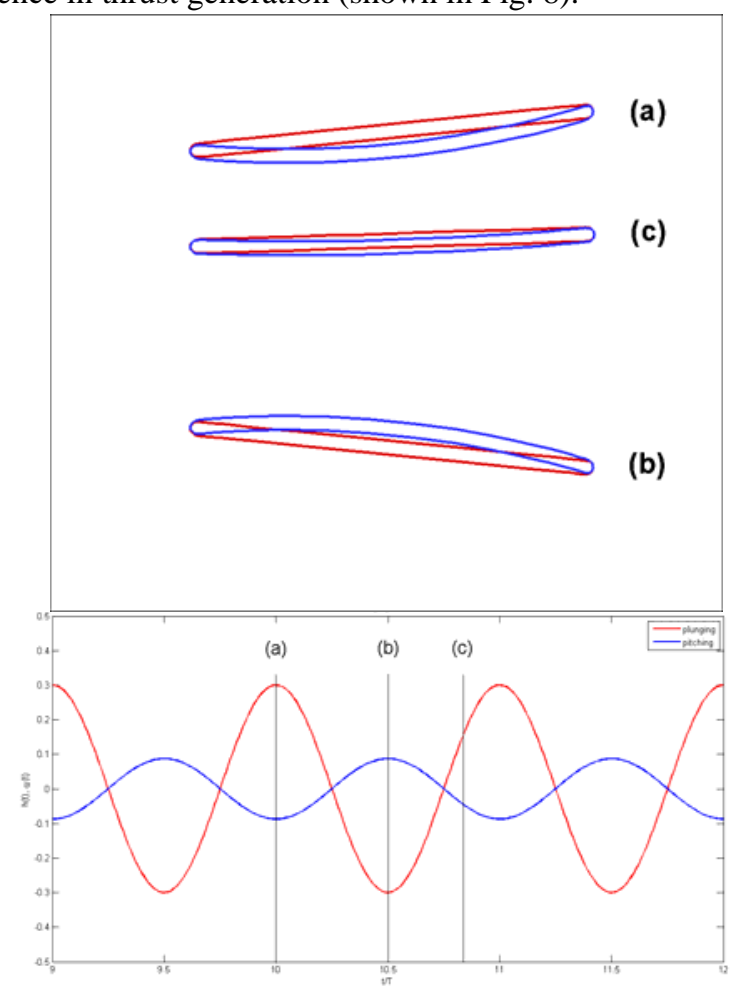

Fig. 8 Shapes at three time instants of the rigid airfoil performing plunging and pitching and prescribed deformation airfoil with only plunging. (red: rigid airfoil; blue: prescribed deformation airfoil)

Fig. 9 presents the streamlines, pressure contours and pressure distribution at the airfoil surface at three time instants in the two cases. Similar to the previous cases presented in Fig. 7, the pressure distributions around the two airfoils at the same time are very comparable. Furthermore, at time instant (a), when the airfoil is plunging downward, the large pressure on the lower surface of the airfoil generate a horizontal component because the airfoil is pitching down and displays a substantial angle of attack. At time instant (b), the situation is reversed. As the airfoil is moving upward, the pressure on the upper surface is much larger than on the lower surface. The pressure generates a horizontal force component on the pitching up airfoil. At time instant (c), the pressure on the upper surface is larger than on the lower surface. As the airfoil is pitching down, backward horizontal component is generated, which explains that there is a small positive part in $\mathrm{C}_{\mathrm{x}}$ time history. 


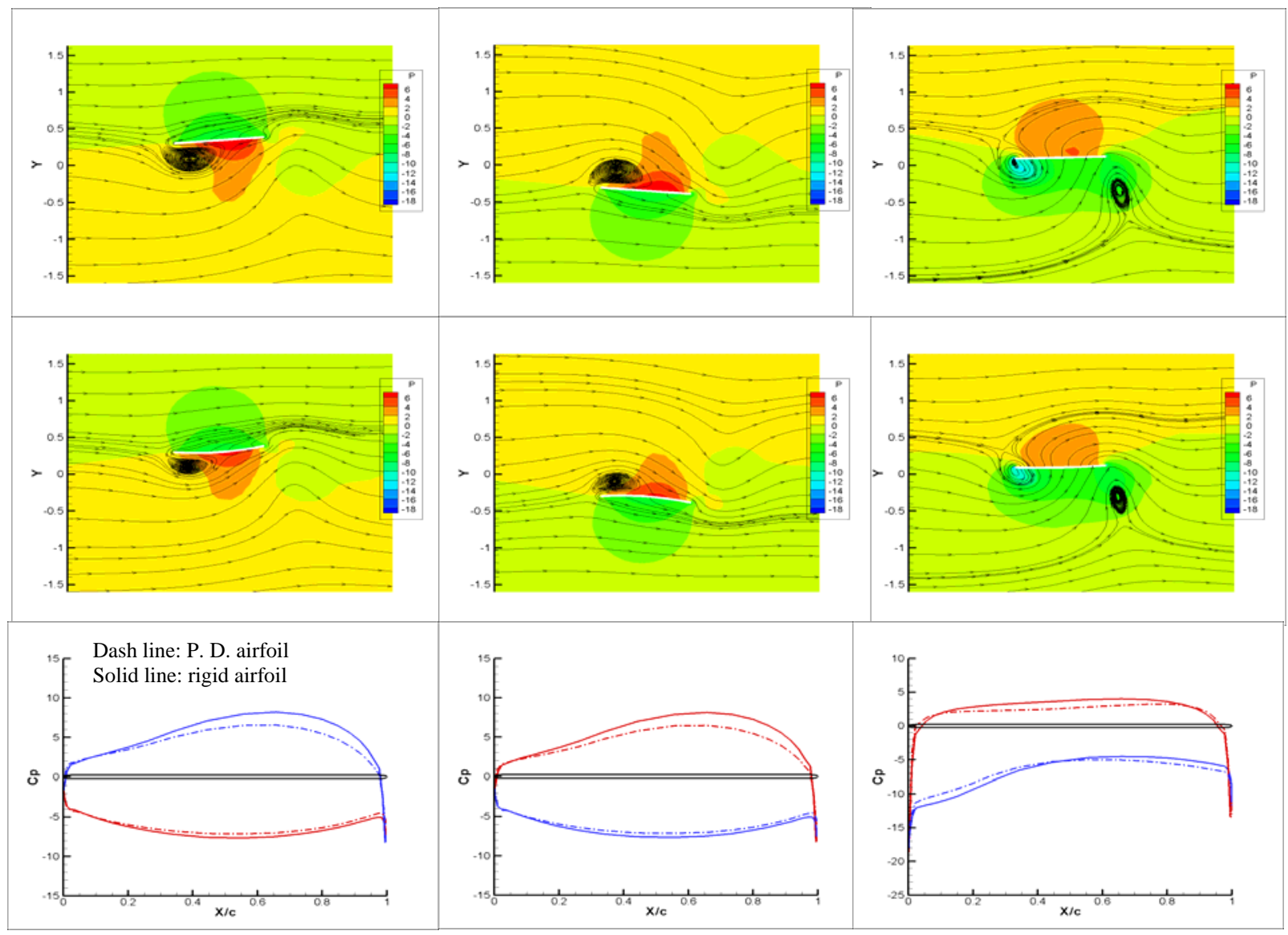

(a)

(b)

(c)

Fig. 9 Streamlines, pressure contours (upper two) and pressure distribution on the airfoil surface (bottom) of the corresponding time instants in Fig. 8 (P. D.: prescribed deformation).

The forces acted on two airfoils are presented in Fig. 10. The computational results indicate that both rigid and the airfoil of prescribed deformation generate comparable forces. Both the forces in $\mathrm{x}$ - and $\mathrm{y}$ direction have same time history pattern. The force peak values are very close to each other in the two cases. Rigid pitching airfoil generates a little larger force in both $\mathrm{x}$ - and $\mathrm{y}$ - direction but not a qualitative difference. This indicates that deformation acted as a passive pitching motion. Considering that in the plunging motion the force acting on airfoil is dominant by pressure, the viscous force is negligible. Based on these two cases, one can further confirm the investigation done in the $R e=9000$ cases, that the differences in thrust largely reflect the differences in the equivalent pitching angle. At least for the range studied here, the detailed shape of an airfoil does not affect the thrust as much as the effective angle of attack. To test this explanation, the y-component of the force is projected to $\mathrm{x}$-direction based on the airfoil's instantaneous pitching angle. The results are shown in Fig. 10. It is clear the projected results are very close to the simulation results, reflecting same time variation and the difference between prescribed deformation and rigid airfoil. 


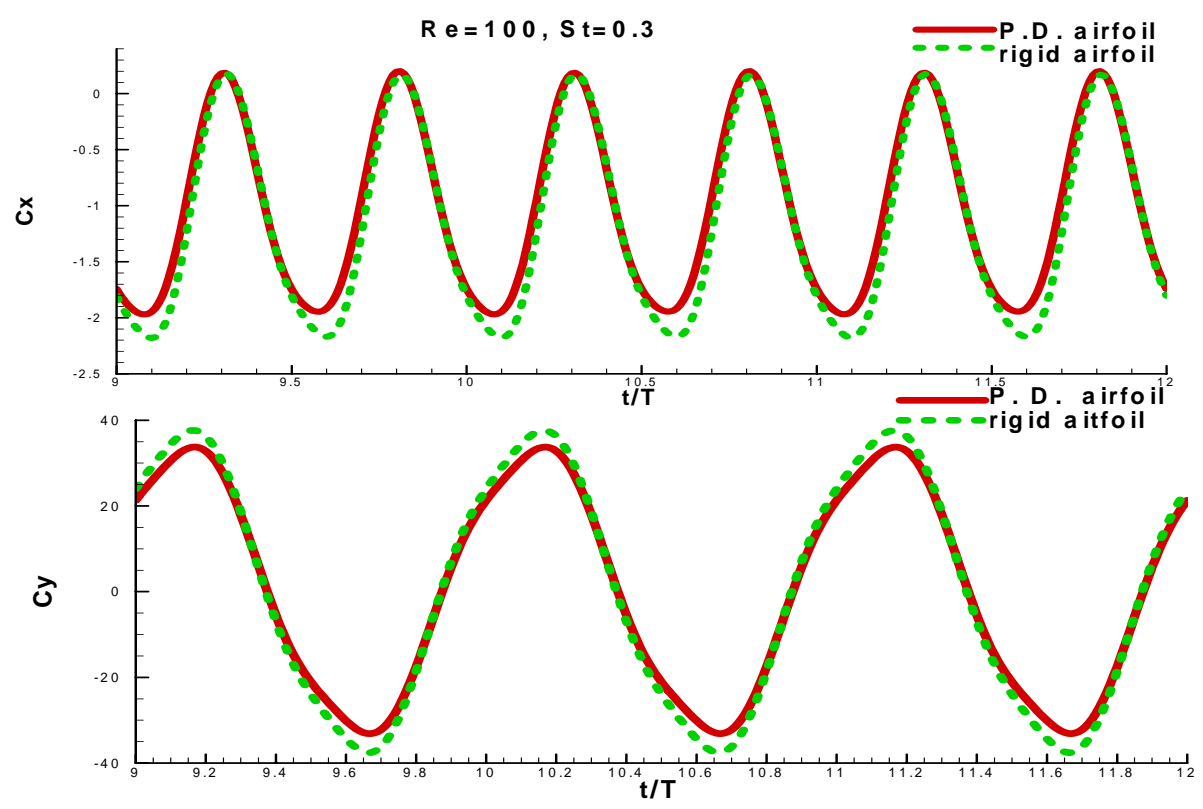

Fig. 10 Numerical results of two comparison cases in study the thrust generation (P.D.: prescribed deformation).

From the above discussion, it seems clear that in response to the aerodynamic loading, a flexible airfoil performs a passive pitching motion to change the effective angle of attack, causing noticeable differences in lift and thrust generation. Furthermore, as evidenced in the case study of the prescribed shape change, even at $\mathrm{Re}=100$, in the plunging motion, the force acting on airfoil is dominated by pressure and the viscous force is of little impact on the overall aerodynamic outcome. Detailed airfoil shape is secondary compared to the equivalent angle of attack.

\section{Conclusion}

Navier-Stokes simulations are employed to research the fluid flow around the flexible plunging airfoils in incoming flow to observe fluid dynamic characteristics and the mechanism in generating thrust. The effect of airfoil flexibility is simulated by coupling a finite element structural solver using beam elements with the fluid solver. Prescribed deformation is used in investigation thrust generation mechanism. Investigation on the computational results indicates:

1. Numerical simulation indicates that the flexible structure causes an equivalent pitching motion. The pitching motion decreases the effective angle of attack. The equivalent pitching motion has a phase angle ahead of the plunging motion, which is consistent with the experimental observation of Heathcote and Gursul $^{18}$.

2. In response to the aerodynamic loading, a flexible airfoil performs a passive pitching motion to change the effective angle of attack, causing noticeable differences in lift and thrust generation. 
3. Within the range of the flexibility considered, the flow fields are similar in all cases. Even at $\mathrm{Re}=100$, in the plunging motion, the force acting on airfoil is dominated by pressure and the viscous force is of little impact on the overall lift and thrust generation. Detailed airfoil shape is secondary compared to the equivalent angle of attack.

Based on the present effort, detailed comparison based on identical flow and structural parameters to those of Heathcote and Gursul ${ }^{18}$ will be conducted next and reported in the near future.

\section{Acknowledgments}

The work presented here has been supported by the US Air Force. We thank Mr. Chang-Kwon Kang for his assistance in processing the computational results. 


\section{References}

${ }^{1}$ Ellington, C.P., "The Aerodynamics of Hovering Insect Flight. I. The Quasi-Steady Analysis," Philosophical Transactions of the Royal Society of London, Series B, Vol. Vol. 305, 1984, pp. 1-15.

${ }^{2}$ Weis-Fogh, T., "Quick Estimate of Flight Fitness in Hovering Animals, Including Novel Mechanisms for Lift Production," Journal of Experimental Biology, Vol. Vol. 59, 1973, pp. 169-230.

${ }^{3}$ Ellington, C.P., Berg, C.v.d., Willmott, A.P., and Thomas, A.L.R., "Leading-Edge Vortices in Insect Flight," Nature, Vol. Vol. 384, 1996, pp. 626-630.

${ }^{4}$ Dickinson, M.H., Lehmann, F.-O., and P.Sane, S., "Wing Rotation and the Aerodynamic Basis of Insect Flight," Science, Vol. 284, 1999, pp. 1954-1960.

${ }^{5}$ Liu, H. and Kawachi, K., "A Numerical Study of Insect Flight," Journal of Computational Physics, Vol. Vol. 146, 1998, pp. 124-156.

${ }^{6}$ Van den Berg, C. and Ellington, C.P., "The Three-Dimensional Leading-Edge Vortex of a 'Hovering' Model Hawkmoth," Phil. Trans. R. Soc. Lond. B, Vol. 352, 1997, pp. 329-340.

${ }^{7}$ Sun, M. and Tang, J., "Unsteady Aerodynamic Force Generation by a Model Fruit Fly Wing in Flapping Motion," Journal of Experimental Biology, Vol. 205, 2002, pp. 55-70.

${ }^{8}$ Ramamurti, R. and Sandberg, W., "A Three-Dimensional Computational Study of the Aerodynamic Mechanisms of Insect Flight," Journal of Experimental Biology, Vol. Vol. 205, 2002, pp. 1507-1518.

${ }^{9}$ Wang, Z.J., Birch, J.M., and Dickinson, M.H., "Unsteady Forces and Flows in Low Reynolds Number Hovering Flight:Two-Dimensional Computations Vs Robotic Wing Experiments," Journal of Experimental Biology, Vol. 207, 2004, pp. 449-460.

${ }^{10}$ Viieru, D., Tang, J., Lian, Y., Liu, H., and Shyy, W., "Flapping and Flexible Wing Aerodynamics of Low Reynolds Number Flight Vehicles," AIAA paper 2006-0503, 2006.

${ }^{11}$ Birch, J. M., Dickson W. B., Dickinson M. H., "Force Production and Flow Structure of the Leading Edge Vortex on Flapping Wings at High and Low Reynolds Numbers," Journal of Experimental Biology, Vol. 207, 2004, pp. 1063-1072.

12 Tang, J., Viieru, D., and Shyy, W., "Effects of Reynolds Number and Stroke Amplitude on Hovering Wing Aerodynamics," 45th Aerospace Sciences Meeting \& Exhibit, AIAA paper 2007-129, 2007. 24.

${ }^{13}$ Freymuth, P., "Thrust Generation by an Airfoil in Hover Modes," Expts Fluid, Vol. 9, 1990, pp. 17-

${ }^{14}$ Shyy, W., Lian, Y., Tang, J., Viieru, D., Liu, H. Aerodynamics of Low Reynolds Number Flyers, Cambridge University Press, New York, to be published.

${ }^{15}$ Lian, Y., Shyy, W., Viieru, D., and Zhang, B.N., "Membrane Wing Aerodynamics for Micro Air Vehicles," Progress in Aerospace Sciences, Vol. 39, 2003, pp. 425-465.

${ }^{16}$ Ifju, P.G., Ettinger, S., Lian, Y., W., S., and Waszak, R.M., "Flexible-Wing-Based Micro Air Vehicles," AIAA paper 2002-0705, Jan. 2002.

${ }^{17}$ Heathcote, S., Martin, D., and Gursul, I., "Flexible Flapping Airfoil Propulsion at Zero Freestream Velocity," AIAA Journal, Vol. 42, No. 11, 2004, pp. 2196-2204.

${ }^{18}$ Heathcote, S., and Gursul, I., "Flexible Flapping Airfoil Propulsion at Low Reynolds Numbers," AIAA Paper 2005-1405, Jan. 2005.

${ }^{19}$ Patankar, S.V. and Spalding, D.B., "A Calculation Procedure for Heat, Mass and Momentum Transfer in Three-Dimensional Parabolic Flows," Int. J. Heat Mass Transfer, Vol. 15, 1972, pp. 1787-1806.

${ }^{20}$ Shyy, W., "Computational Modeling for Fluid Flow and Interfacial Transport," edited, Amsterdam, The Netherlands, Elsevier. 1994. 
${ }^{21}$ Thomas, P.D. and Lombard, K., "Geometric Conservation Law and Its Application to Flow Computations on Moving Grids," AIAA Journal, Vol. 17, 1979, pp. 1030-1037.

${ }^{22}$ Kamakoti, R. and Shyy, W., "Fluid-Structure Interaction for Aeroelastic Applications," Progress in Aerospace Sciences, Vol. 40, 2004, pp. 535-558. 